\title{
Heavy Flavour Production at HERA
}

\author{
Christoph Grab \\ a Institute of Particle Physics, \\ ETH Zurich, CH-8093 Zurich, Switzerland
}

On behalf of the H1 and ZEUS collaborations

An overview of new measurements of heavy flavour production in $e-p$ collisions done by the H1 and ZEUS experiments at HERA is presented. Various techniques are used to efficiently tag the heavy flavour in the events, thereby exploring different regions of phase space. Differential cross sections are measured both in photoproduction and in deep inelastic scattering. The predictions based on perturbative QCD calculations at next-to-leading order are found to generally agree with the charm production data, but to lie somewhat below the beauty data.

\section{Introduction}

Measuring charm and beauty production in ep collisions at HERA is ideally suited to study the strong force. The dominant production mechanism is the photon-gluon-fusion process, directly driven by the gluon density in the proton. The large mass of the heavy quark, $m_{q}$, provides a hard scale, which allows calculations by means of perturbative QCD. Additional hard scales, such as the virtuality $Q^{2}$ of the exchanged photon or the transverse momenta of the outgoing quarks, complicate the picture. Experimentally, the measurements are performed both in the region of photoproduction $\left(\gamma p\right.$ with $\left.Q^{2} \approx 0 \mathrm{GeV}^{2}\right)$ and in deep inelastic scattering (DIS with $2<Q^{2}<100$ $\left.\mathrm{GeV}^{2}\right)$. At the scales $\mathrm{O}\left(m_{q}\right)$ considered here, pQCD calculations performed in the "massive scheme" at Next-to-Leading Order (NLO) are expected to give reliable results. Consequently, the data are compared with NLO predictions in the "massive scheme" ([1,2]).

\section{Measurements of charm}

Measuring the $\mathrm{D}^{*}$ production cross section as a function of $\mathrm{Q}^{2}$ is a stringent test of the NLO calculation for charm production in the whole region from $\gamma \mathrm{p}$ to DIS. The cross section measured by the ZEUS collaboration is well described by the predictions of NLO QCD (figure 1) over the full four orders of magnitude, showing that this large kinematic region is well understood theoretically.

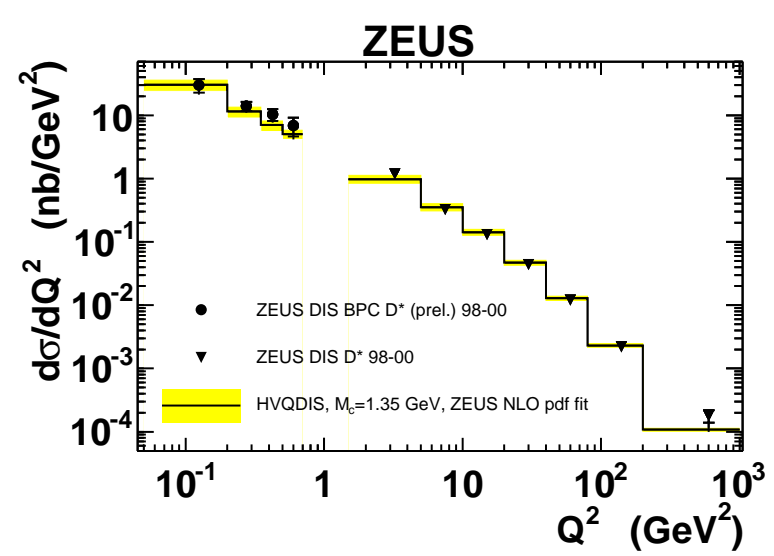

Figure 1. Differential D* cross section as a function of $\mathrm{Q}^{2}$.

For events containing a $\mathrm{D}^{*}$ and two jets in $\gamma \mathrm{p}$, comparisons of both jets and $D^{*}$ momentum spectra (see figure 2) with pQCD calculations yield very good agreement[3]. In addition, correlations can be studied between the jets and allow detailed comparisons with QCD calculations [4]. $\mathrm{x}_{\gamma}^{\mathrm{obs}}$ is the fraction of the photon's four momentum at LO, entering the hard dijet subprocess, which is measured with the two highest $\mathrm{p}_{\mathrm{T}}$ jets.

The NLO calculation describes the shape of the 

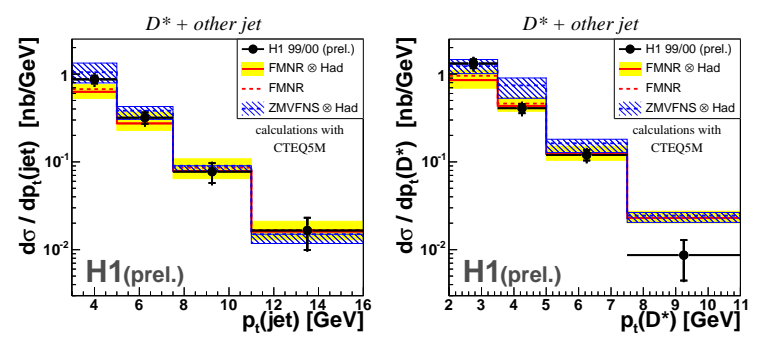

Figure 2. Cross section $\mathrm{d} \sigma / \mathrm{d} p_{T}$ for $\mathrm{D}^{*}$ and 2 nd jet in photoproduction.

data for direct $\gamma \mathrm{p}\left(\mathrm{x}_{\gamma}^{\mathrm{obs}}>0.75\right)$ pretty well. But, the shape is not as well described by the calculation for resolved $\gamma \mathrm{p}\left(\mathrm{x}_{\gamma}^{\mathrm{obs}}<0.75\right)$ data, indicating a need for higher order corrections to the NLO calculations. The LO + parton shower Monte Carlo simulations, particularly HERWIG, fit the data shape well for both direct and resolved $\gamma \mathrm{p}$ (figure 3), but fall short in terms of normalisation. Similar results have been found looking at correlations between the $\mathrm{D}^{*}$ and a jet not containing the $\mathrm{D}^{*}[3]$.

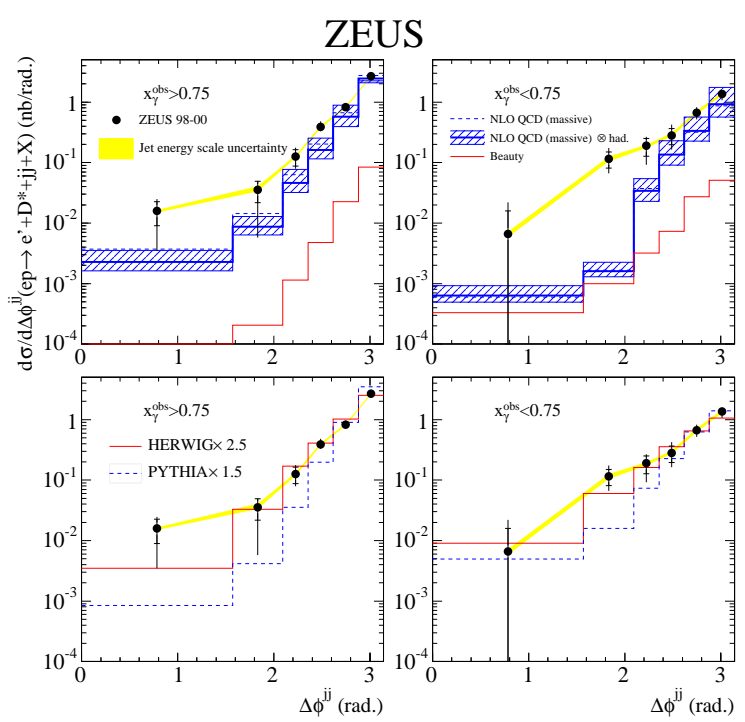

Figure 3. Cross section $\mathrm{d} \sigma / \mathrm{d} \Delta \phi j j$ for dijets in $\mathrm{D}^{*}$ photoproduction.

\section{Measurements of beauty}

To separate $b$ from $c$ and light quark background, the large mass and the long lifetime of the b-quark are exploited. In practice, the following variables are used: 1) $p_{T}^{r e l}$ : The relative transverse momentum of the muon with respect to the axis of an associated jet in semileptonic decays; 2 ) the impact parameter $\delta$ : the distance of a track in the transverse plane with respect to the ep collision vertex, where $\delta$ can be precisely measured with the silicon track detectors $[5,6] ; 3$ ) $S=\delta / \sigma_{\delta}, S_{1}, S_{2}$ : the impact parameter significances of tracks (ordered according to size) in the event. The fractions of $\mathrm{c}$ - and b-quark events
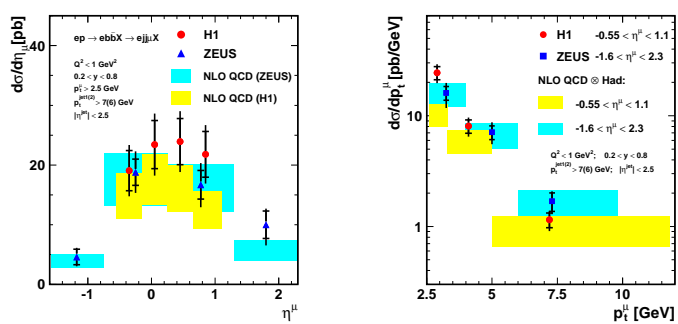

Figure 4. Differential cross section for the process $e p \rightarrow e b \bar{b} X \rightarrow e j j \mu X^{\prime}$ as functions of the muon variables pseudo-rapidity $\eta_{m u}$ and transverse momentum $p_{t}^{\mu}$. a) and b) show the H1 and ZEUS results and the NLO predictions in photoproduction.

in the data samples are then determined from fits to distributions of $p_{T}^{r e l}, \delta, S_{i}$ and/or combinations thereof. Properly normalising the fractions yields directly the $b$-production cross sections, using the different tagging techniques as described below.

(i) Tagging beauty with muons and jets: In these analyses $b$-events are identified using muons from semileptonic b-decays, which provide a clean experimental signature. In addition to the muon the presence of at least one jet (two jets) in the DIS (photoproduction) sample is required. Figure 4 shows the differential cross-sections measured by H1 [7] and ZEUS [8] in photoproduction as a function of the muon pseudorapidity (left) and transverse momentum (right). Both 
measurements agree well in the overlapping region. Also shown are NLO pQCD predictions[1], in which the fragmentation is performed using the Peterson function. The errors of the theory prediction (bands) are dominated by the scale uncertainties. With a tendency to lie below the data, this calculation describes the $\mathrm{H} 1$ and ZEUS data points within the uncertainties. In the lowest $p_{T}$ bin the H1 measurement somewhat exceeds the prediction while at higher transverse momenta a better agreement is observed. Such an excess is not seen in the ZEUS data. Their new silicon microvertex detector (MVD) allowed ZEUS to complement previous measurements using the latest HERA-II data. Their results are found to be compatible with the previous ZEUS measurements and with NLO QCD. In the DIS regime, the $\mathrm{H} 1$ and ZEUS measurements are done in similar kinematic regions. The observations lead to conclusions very similar to the photoproduction case.

(ii) Tagging beauty by lifetime information: The method, applied in photoproduction, exploits explicitly the large lifetime information and it relies only on the significances $S_{i}([9])$. Without requiring a detected muon it thus extends the phase space of $b$ transverse momenta. While the charm data are reasonably well described in both normalization and shape, the beauty data are again found to be somewhat higher than the NLO prediction.

(iii) Double tagging beauty by $D^{*}$ and muons: Further measurements by H1 ([10]) and ZEUS ([11]) are based on the simultaneous detection of a $D^{* \pm}$ meson and a muon. The charge and angular correlation between the two serves to separate the $b$ and $c$ contributions. The kinematic region for $b \bar{b}$ is characterised by lower $b \bar{b}$ centre-of-mass energies than in most previous analyses, which require high momentum jets. A shape comparison of the measured differential distributions with QCD calculations at LO and NLO clearly indicate that effects beyond the LO approximation are directly observed. Again, charm is better described by NLO QCD than is beauty, where the differences are largest at low $p_{t}(\mu)$ and for more forward $\eta_{t}(\mu)$.

(iv) Double tagging beauty by two muons: A con- ceptually very similar analysis by ZEUS [12] measures events in which two muons are observed in the final state. A low $p_{t}(\mu)$ threshold in combination with a large rapidity coverage accesses essentially the full phase space for $b$-production. The differential cross sections are shown in Figure 5a) and $\mathrm{b}$ ). The LO QCD predictions, based on the Pythia and Rapgap Monte Carlo programs, describe the shapes pretty well, but are a factor of two low in normalisation.

When comparing the total inclusive cross sections, which covers the $b$-phase space down to $p_{t}(b) \approx 0$, it is found, that the NLO pQCD predictions are a factor of roughly 2.4 below the measurements, although they are still compatible within the very large uncertainties.
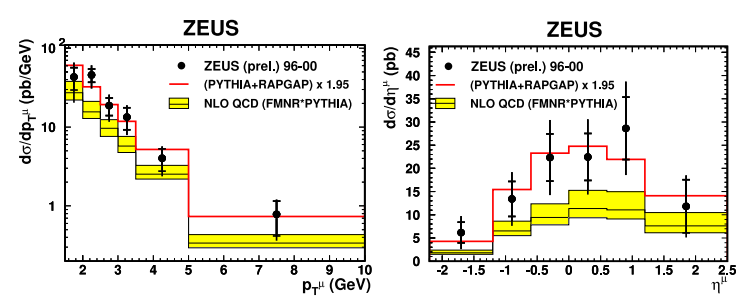

Figure 5. a) and b) show the differential cross sections for $e p \rightarrow e b \bar{b} X \rightarrow e \mu \mu X^{\prime}$ measured by ZEUS in double muon tagging in photoproduction. Overlaid are the LO predictions scaled up by a factor of 1.95 .

\section{Heavy Quark structure functions}

By using the impact parameter significance of tracks, charm and beauty fractions are extracted by fitting distributions in different $\mathrm{x}-\mathrm{Q}^{2}$ intervals $[13,14]$. Then the differential cross sections are measured and the structure functions $\mathrm{F}_{2}^{\mathrm{c} \overline{\mathrm{c}}}$ and $\mathrm{F}_{2}^{\mathrm{b} \overline{\mathrm{b}}}$ calculated from the expression

$$
\frac{\mathrm{d}^{2} \sigma^{b \bar{b}}}{\mathrm{~d} x \mathrm{~d} Q^{2}}=\frac{2 \pi \alpha^{2}}{x Q^{4}}\left[\left(1+(1-y)^{2}\right) F_{2}^{b \bar{b}}-y^{2} F_{L}^{b \bar{b}}\right]
$$

The $\mathrm{F}_{2}^{\mathrm{b} \overline{\mathrm{b}}}$ is plotted in figure 6. The QCD calculations fit the data reasonably well with scaling violations apparent at low $\mathrm{x}$. 


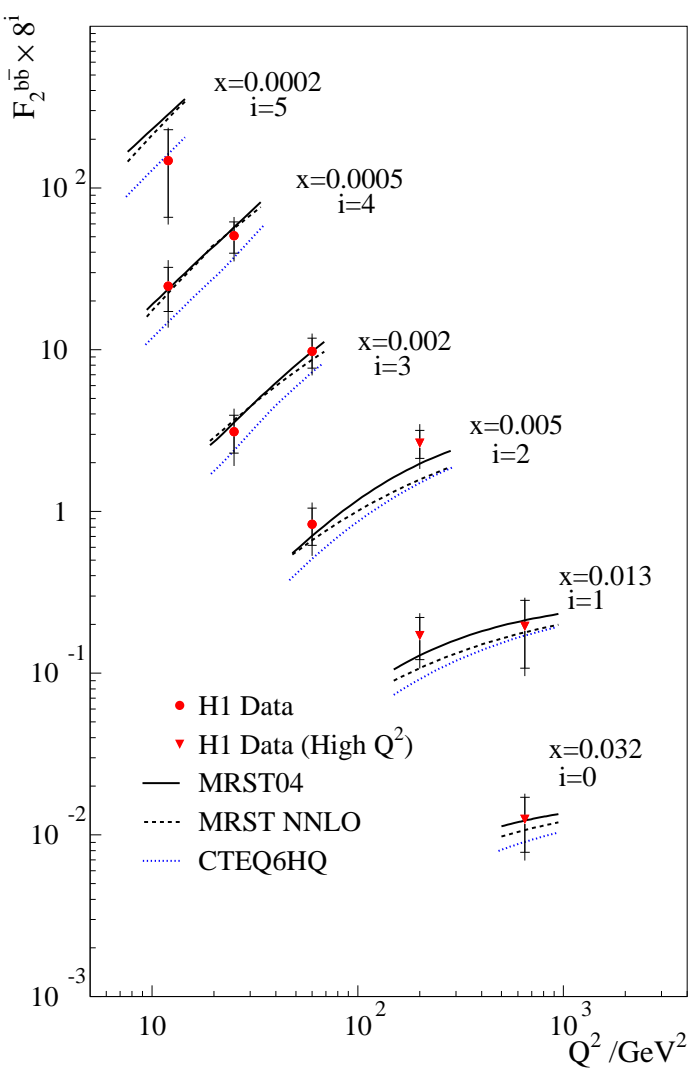

Figure 6. Beauty structure function $F_{2}^{b \bar{b}}$ as a function of $\mathrm{Q}^{2}$ for various $x$ values.

\section{Summary}

In general the charm production appears to be pretty well described by the perturbative QCD calculations at NLO, although the need for higher-order corrections becomes visible.

The recent inclusive beauty measurements at HERA are summarised in figure 7 , which shows the ratio of measured cross sections to the NLO QCD prediction as a function of $Q^{2}$. Most of the data points lie above the predictions and there is no clear $Q^{2}$-dependence visible. The differential spectra indicate that the data tend to lie above the predictions more significantly towards small b-quark transverse momenta and in the more for- ward (i.e. proton) direction. The single significances are not that large, however they are observed in different regions with differing measuring methods. Results in the DIS regime show somewhat better agreement with the NLO predictions. It is expected that the new HERA II data will provide higher precision. But to resolve the issues completely, improvements are needed also on the theoretical side.

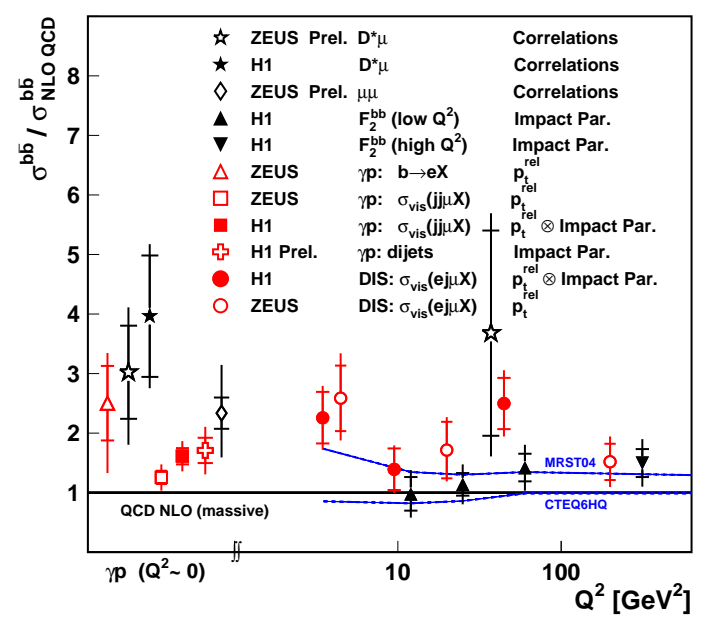

Figure 7. Ratio of the different beauty cross section measurements to the pQCD NLO prediction as a function of $Q^{2}$.

\section{REFERENCES}

1. S. Frixione, P. Nason and G. Ridolfi, Nucl. Phys. B $\mathbf{4 5 4}$ 3 (1995).

2. B.W.Harris and J.Smith, Nucl. Phys. B 452, 109 (1995).

3. A. Aktas et al. [H1 Collab.], Accepted by Eur. Phys. J.C , 05/06; [arXiv:hep-ex/0605016]

4. ZEUS Coll., S. Chekanov et al., Nucl. Phys. B 729492 (2005).

5. D. Pitzl et al., Nucl. Instrum. Meth. A 454 (2000) 334.

6. E. N. Koffeman [ZEUS Collab.], Nucl. Instrum. Meth. A 473, 26 (2001).

7. A. Aktas et al. [H1 Collab.], Eur. Phys. J. C 41, 453 (2005)

8. S. Chekanov et al. [ZEUS Collab.], Phys. Rev. D 70 (2004) 012008 .

9. A. Aktas et al. [H1 Collab.], [arXiv:hep-ex/0507081].

10. A. Aktas et al. [H1 Collab.], Phys. Lett. B 621, 56 (2005).

11. S. Chekanov et al. [ZEUS Collab.], Contributed paper 575 to Int. Conf. on High Energy Physics, 2003, Aachen.

12. S. Chekanov et al. [ZEUS Collab.], Contrib. paper 269 to Int. Conf. on Lepton-Photon interactions, 2005, Uppsala.

13. H1 Coll., A. Aktas et al., Eur. Phys. J. C45 23 (2006).

14. H1 Coll., A. Aktas et al., Eur. Phys. J. C40 349 (2005). 